\title{
Chromatic Colors Were in Vogue in 2020
}

\author{
2020 was without doubt an unusual year. And there was also a surprising shift in the range of colors \\ used in automotive OEM coatings.
}

The BASF Color Report 2020 for Automotive OEM Coatings shows that the range of colors used in the automotive sector is changing in an unconventional way, with a wider range of colorful hues rolling off production lines around the world. However, various colors such as blue and yellow are gaining ground in some regions, while red and violet are slowly closing the gap to achromatic colors in other parts of the world. The expanding color spaces make the overall spectrum broader than 2019 and add a flash of brilliance.
Despite this shift towards more chromatic colors, the achromatic colors of black, white, silver and gray were still popular, and the majority of new vehicles were painted in these colors. White remains the world's most popular vehicle color. It has a classic, timeless beauty, and a connection to both the environment and high technology.

The data published in the report is based on a lower total production output of vehicles compared to the previous years, as the survey includes the period in which car- makers had to shut down production due to Covid-19.

\section{EMEA: greater variety of chromatic colors and tones}

Europe, the Middle East and Africa (EMEA) followed the worldwide trend towards chromatic colors. In 2020, $11 \%$ of new vehicles in the EMEA region were painted blue, thus making blue the most popular chromatic color. The number of vehicles painted in violet also increased significantly, thereby

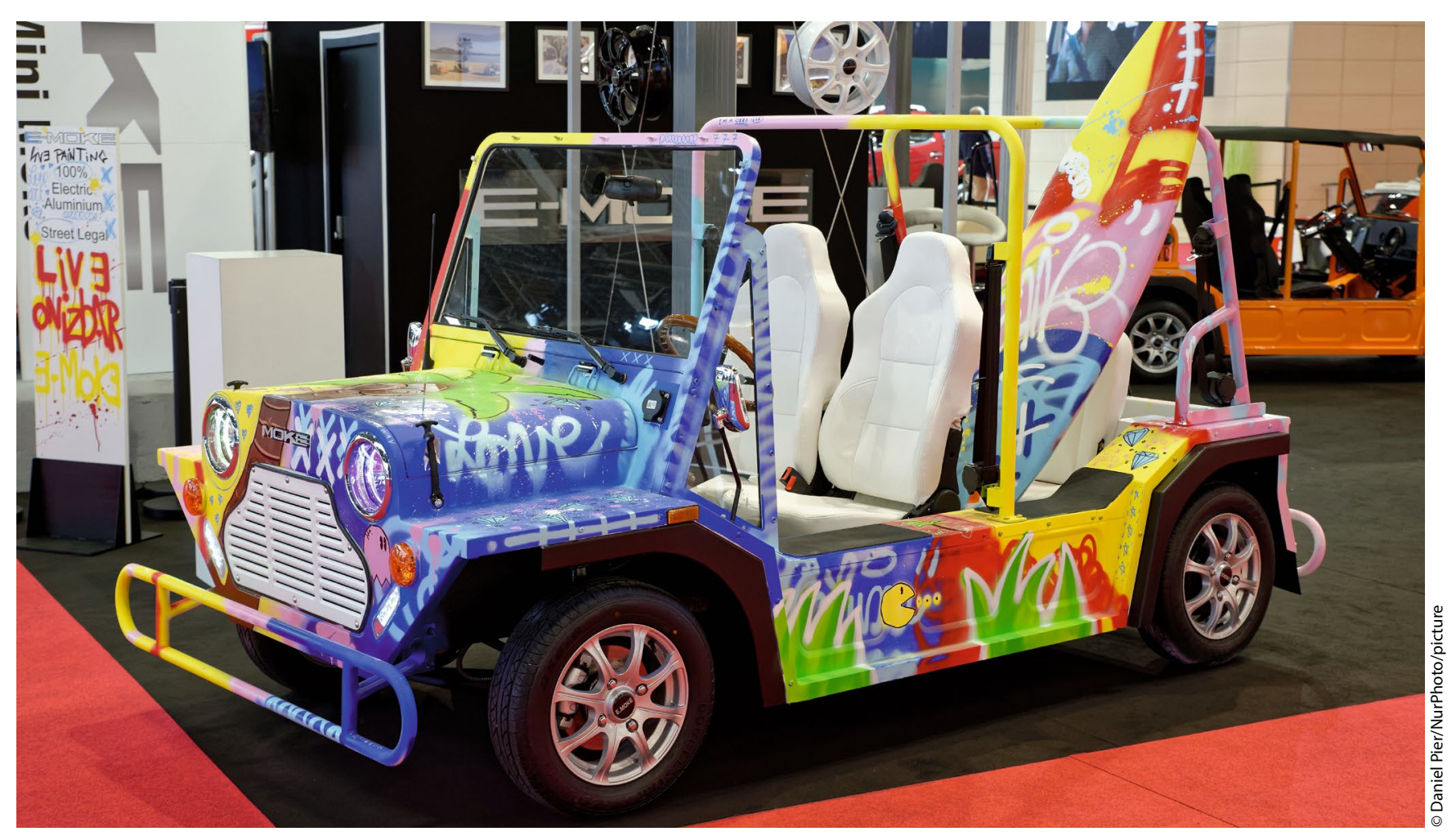

A slight color shift was noticed in vehicle coatings in 2020: Around the world, vehicles rolled off production lines painted in a wider range of chromatic colors. 
The expanding color spaces make the overall spectrum broader than 2019 and add a flash of brilliance.
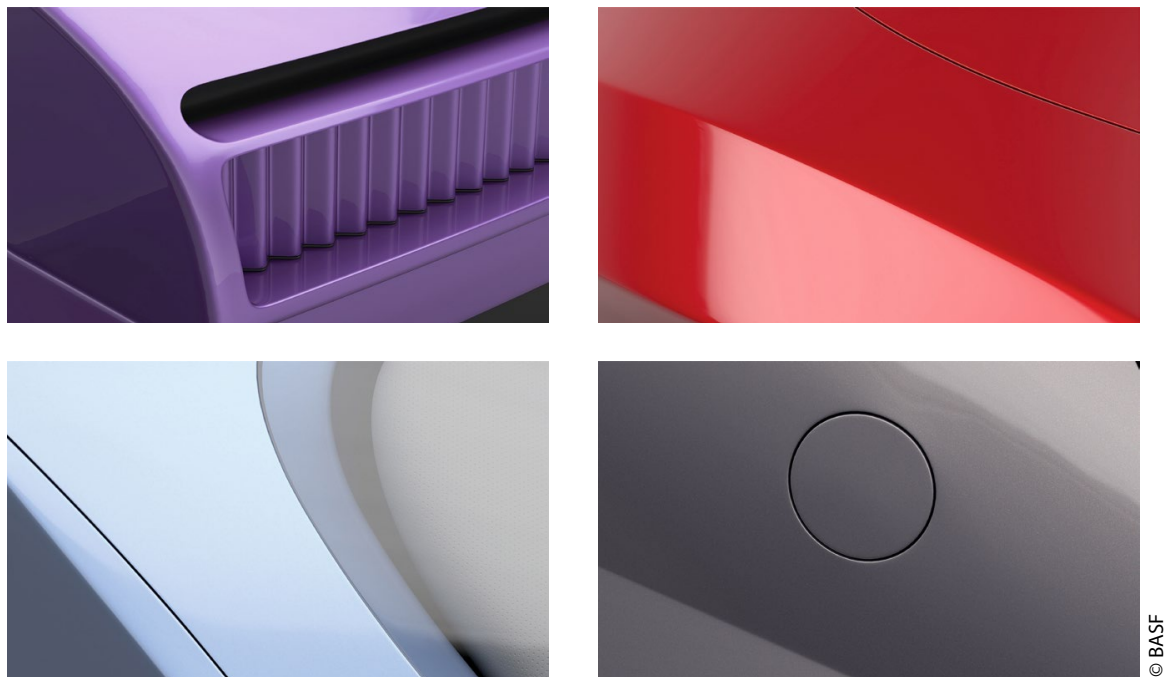

increasing the color diversity further. Other chromatic colors also gained in popularity, particularly for small SUVs, a market segment that continues to grow.

This diversity is due in part to the wide spectrum of color shades. In 2020, carmakers used more than 160 different shades of blue to paint their vehicles in the EMEA region. Gray followed in second place with 140 different hues. Both color spaces were more diverse than white, which had only 70 different tones. In the achromatic spectrum, white colors still lead the market with a share of $28 \%$, ahead of both gray and black.

„Color variety changes with size. For example, while violet has a unique home in mid-size SUVs, it does not appear in great numbers in smaller or larger SUVs," said Mark Gutjahr, head of Automotive Color Design EMEA. „The opposite is true with yellow, which shows up in both the very small and the very large, but not the middle. These are specific and unique color positions that appear and disappear throughout the segments."

\section{North America: blue increasing in popularity}

North American car buyers have a smaller selection of chromatic colors, but they do not purchase fewer chromatic cars, vans or SUVs. Blue has increased in popularity as a vehicle color, overtaking red, while beige and brown have almost completely disappeared from the market. Therefore, green is the only other chromatic color that is used for an appreciable number of vehicles in the region. As early as 2016, the designers at BASF designated blue as ,an important color theme for the automobile industry that will increase its market share over the coming years." Automobile designers often look three to four model years into the future when developing colors.

\section{Asia Pacific: black and gray pick up pace}

The Asia Pacific region is the home of the largest automotive production volume worldwide and is a microcosm of global color popularity. While every area is different, the preferences in the Asia Pacific region reflect global data. The luminous colors mirror the increasing popularity of chromatic colors that can also be observed elsewhere. White remains the most popular color in the region and is used on around $48 \%$ of new vehicles. Black and gray continue to gain in popularity, following a three-year trend which is weakening the lead of white hues. The total number of brown, green and violet vehicles may not be high, but these colors enjoy consistent popularity.

\section{South America: conservative purchasers select more conservative colors}

Colors like red and blue that are popular in other regions around the world also remain in demand in South America, particularly amongst car buyers who attempt to express their individuality with sportier cars. The market preference for achromatic colors is even more remarkable.
岕

Traditionally, South American consumers choose rather conventional, conservative colors. Like in other regions, white is by far the favorite and was used in around 39\% of new vehicles. The popularity of black and silver is higher than in other regions, with a respective share of $18 \%$. With a share of $12 \%$, black is the least popular of the achromatic colors. In total, achromatic colors make up $87 \%$ of vehicle colors.

In contrast to other regions, red stands out as the most popular chromatic color with a share of $9 \%$. Various shades of blue make up a significant proportion around the globe, but only account for $2 \%$ of the market in South America. Orange is a newcomer here, brown is remaining consistent, and beige has almost entirely disappeared. "Trends that are popular in other parts of the world take longer to establish themselves in South America. While the culture in the continent has many colorful aspects, the people tend to be a little more conservative when it comes to their vehicles," said Marcos Fernandes, head of Regional Business Management, Automotive OEM Coatings Solutions, South America. //

\section{Contact}

BASF Coatings GmbH

Münster (Germany)

www.basf-coatings.com 